\section{Case Reports in Ophthalmology}

\title{
A Mystery of Bilateral Annular Choroidal and Exudative Retinal Detachment with No Systemic Involvement: Is It Part of Vogt-Koyanagi-Harada Disease Spectrum or a New Entity?
}

\author{
Ibrahim Elaraoud Walter Andreatta Li Jiang Kenan Damer \\ Jalil Al-Ibrahim \\ Birmingham Midlands Eye Centre, Birmingham, UK
}

\section{Keywords}

Choroidal detachment $\cdot$ Exudative retinal detachment $\cdot$ Vogt-Koyanagi-Harada disease

\begin{abstract}
A 68-year-old Caucasian male presented to the eye emergency department with bilateral significant visual loss. He was otherwise healthy with no significant past medical history. Ophthalmic history was significant for chronic open-angle glaucoma, for which the patient was using latanoprost once daily to both eyes. There was no preceding history of trauma or ocular surgery and the patient was emmetropic. Two weeks prior to his presentation, he reported a headache, which settled spontaneously. Slit-lamp examination demonstrated bilateral keratic precipitates, bilateral significantly shallow anterior chamber, and bilaterally normal intraocular pressures of $16 \mathrm{~mm} \mathrm{Hg}$. Fundal examination was significant for bilateral 360degree choroidal detachments with exudative retinal detachment involving the maculae. These findings were confirmed using wide-field fundus photography, B-scan ultrasonography, and optical coherence tomography. Fundus fluorescein angiography did not reveal any evidence of retinal vasculitis. Indocyanine green chorioangiography of the posterior pole showed multiple areas of focal choroidal hypoperfusion. Extensive systemic investigation
\end{abstract}


demonstrated no infectious, neoplastic, or inflammatory cause, and the patient did not complain of any systemic symptoms. Treatment with high-dose intravenous methylprednisolone was administered and this brought about complete resolution of both choroidal and retinal detachments, with partial visual recovery.

\section{Introduction}

The differential diagnosis of choroidal detachment (CD) associated with exudative retinal detachment (ERD) includes Vogt-Koyanagi-Harada disease [1] (VKH), uveal effusion syndrome [2], metastases [3], and posterior scleritis [4]. VKH is a systemic condition which is associated with a granulomatous pan-uveitis. VKH usually affects pigmented races and the incidence of this condition varies greatly, from 1-4\% of all uveitis patients in the United States to $6.8-9.2 \%$ of similar patients in Japan [5]. The etiology of VKH is not fully understood; however, it is believed to be due to a T-cell-mediated immune response to melanin, retinal pigment epithelium, and melanocytes, which leads to both ocular and systemic involvement [6].

Ocular manifestations include choroiditis with ERD and in severe cases CD has been reported. In 2001, the First International Workshop on Vogt-Koyanagi-Harada Disease proposes diagnostic criteria that rely on specific ocular and systemic criteria. Ocular criteria include no history of ocular trauma or surgery, no other clinical or laboratory findings to explain the presentation, the presence of subretinal fluid, and focal areas of choroidal hypoperfusion.

The systemic manifestations include meningism and tinnitus. Alopecia, poliosis, and vitiligo usually are late signs. It is worth noting that isolated headaches are not considered to be part of the criteria. Due to its inflammatory nature VKH usually responds remarkably well to systemic steroids and immune modulators.

Uveal effusion syndrome is most common in nanophthalmic eyes and hypermetropic eyes with thickened sclera. It is not normally associated with intraocular inflammation and responds poorly to systemic steroid. Both sclerotomy with drainage of fluid, and vitrectomy have been documented as management options [4].

Posterior scleritis is normally painful and depending on the cause, might have other ocular involvement, leading to both CD and ERD. B-ultrasonography is helpful in confirming the diagnosis and this condition tends to respond well to immunosuppression.

Metastasis and masquerade syndromes are another rare cause of CD and ERD. Such lesions may present diagnostic challenges and multiple investigation modalities including fundus fluorescein angiography, B-ultrasonography, and ocular biopsy may be needed in order to confirm this.

\section{Case Report}

A 68-year-old Caucasian male who was otherwise fit and healthy, presented to the emergency ophthalmology unit as he had noticed a bilateral, gradual, painless loss of vision over the past 2-3 weeks. Prior to this, he reported that he had experienced a mild headache which had settled with simple analgesia. The patient denied malaise, nausea, vomiting, neck stiffness, or abdominal pain. On presentation, his Snellen visual acuity was 6/60 in the right eye and hand movement in the left. Intraocular pressure was $16 \mathrm{~mm} \mathrm{Hg}$ in both eyes. Slit- 
lamp examination of the anterior segments showed granulomatous keratic precipitates with significant shallowing of the anterior chambers, mild anterior chamber cell activity, and few areas of posterior synechiae (Fig. 1). There was evidence of a nonsignificant cataract and mild vitritis. Dilated fundus examination showed extensive annular CD and ERD with no signs of clinical vasculitis or retinitis (Fig. 2). Optic coherence tomography scans showed bilateral extensive subretinal fluid at the left macula and less so at the right (Fig. 3).

B-scan ultrasonography confirmed extensive choroidal detachments in 4 quadrants with no associated scleral thickening or masses affecting the choroid or sclera (Fig. 4). Ultrasound biomicroscopy confirmed shallow anterior chambers with no ciliary body mass or other pathology in either eye. Indocyanine green chorioangiography showed multiple areas of choroidal hypoperfusion (Fig. 5). Fundus fluorescein angiography showed pooling of fluorescein in late frame in the subretinal space (Fig. 2). The patient was admitted and underwent urgent chest X-ray and blood tests to investigate for the presence of possible infections or inflammatory causes. His chest X-ray was normal; however, inflammatory markers were high (ESR and CRP). An inflammatory etiology was suspected and the patient was treated with high-dose intravenous prednisolone $1 \mathrm{~g}$ of intravenous methylprednisolone for 3 days).

A good response was observed and both CD and ERD were noted to resolve over a period of 1 week. The patient was further investigated with MRI of the brain, and CT scans of the thorax, abdomen, and pelvis to exclude possible malignancy or inflammation. Detailed hematological, immunological, microbiological, and biochemical workup failed to identify any specific etiology to explain his condition.

Following intravenous methylprednisolone, treatment was continued with a slowly tapering course of oral prednisolone. Four weeks after his initial presentation, visual acuity was observed to have improved in both eyes, to 6/18 in the right eye and 6/36 in the left. Anterior chamber depth was restored and the retina remained flat. Autofluorescence images highlighted bilateral macular retinal pigment epithelium changes, more prominent in the left eye with obvious damage to photoreceptor/retinal pigment epithelium complex (Fig. 3), which could explain the limited visual recovery.

\section{Discussion}

This patient has proved to be a diagnostic challenge, as he did not fit clearly in any of the categories that are known to cause an extensive CD with ERD. Uveal effusion syndrome tends to occur in smaller eyes with abnormal sclera, which our patient did not have, and granulomatous inflammation picture goes against it. There was no pain and B-ultrasonography showed no signs of posterior scleritis or scleral or choroidal masses of note. The patient did not meet the defined diagnostic criteria for VKH. Headache alone is not sufficient to meet the definition of meningism as qualified by the revised Diagnostic Criteria for VKH [7]. In view of his age, ethnicity and the extensive bilateral anterior choroidal detachment and absence of systemic symptoms, the diagnosis of VKH is not fully supported.

On the other hand, he did manifest some clinical signs which favor VKH as a potential diagnosis, including the granulomatous panuevitis with areas of focal choroidal hypoperfusion on indocyanine green chorioangiography and the presence of subretinal fluid involving the posterior pole. The remarkably quick response to high-dose steroid is also well recognized in VKH. 


\section{References}

1 Yamamoto N, Naito K: Annular choroidal detachment in a patient with Vogt-Koyanagi-Harada disease. Graefe's Arch Clin Exp Ophthalmol 2004;242:355-358.

$\longrightarrow 2$ Kreiger AE, Meyer D, Smith TR, Riemer K: Metastatic carcinoma to the choroid with choroidal detachment: a case presenting as uveal effusion. Arch Ophthalmol 1969;82:209-213.

-3 Elagouz M, Stanescu-Segall D, Jackson TL: Uveal effusion syndrome. Surv Ophthalmol 2010;55:134145.

4 Rudd JC: 49 year old man with 6 months of swelling around the right eye. Digit J Ophthalmol 1998;4:20. 5 Agarwal A: Gass's Atlas of Macular Diseases, 5th ed. China, Saunders, 2012, pp 998-1002.

-6 Attia S, Khochtali S, Kahloun R, Zaouali S, Khairallah M: Vogt-Koyanagi-Harada disease. Expert Rev Ophthalmol 2012;7:565-585.

-7 Read RW, Holland GN, Rao NA, et al: Revised diagnostic criteria for Vogt-Koyanagi-Harada disease: report of an international committee on nomenclature. Am J Ophthalmol 2001;131:647-652.
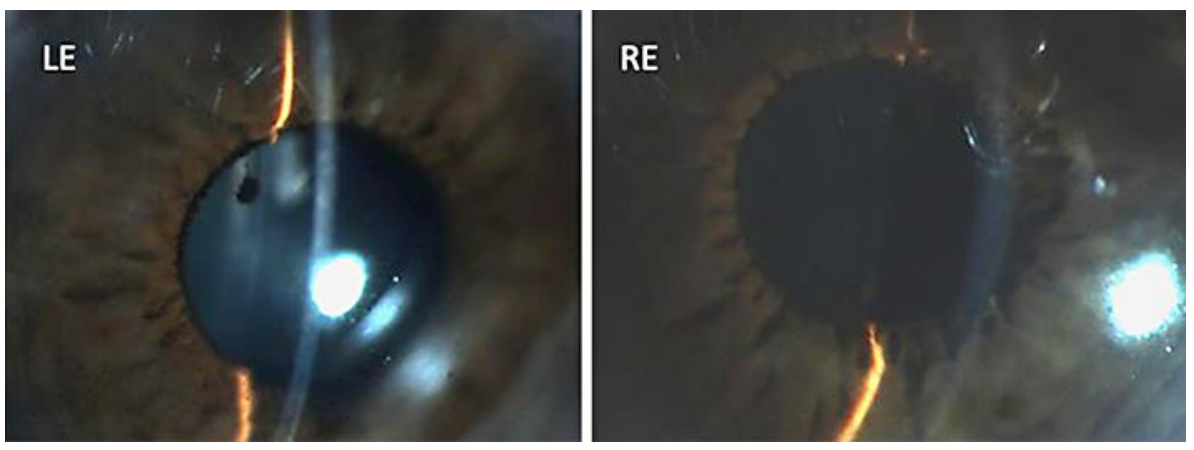

Fig. 1. Both eyes demonstrate a shallow anterior chamber with elements of posterior synechiae and keratic precipitates more obvious in the left eye. 


\section{Case Reports in Ophthalmology}

\begin{tabular}{l|l}
\hline Case Rep Ophthalmol 2017;8:1-6 \\
\hline DOI: 10.1159/000453543 & $\begin{array}{l}\text { @ 2017 The Author(s). Published by S. Karger AG, Basel } \\
\text { www.karger.com/cop }\end{array}$ \\
\hline
\end{tabular}

Elaraoud et al.: A Mystery of Bilateral Annular Choroidal and Exudative Retinal Detachment with No Systemic Involvement: Is It Part of Vogt-Koyanagi-Harada Disease Spectrum or a New Entity?
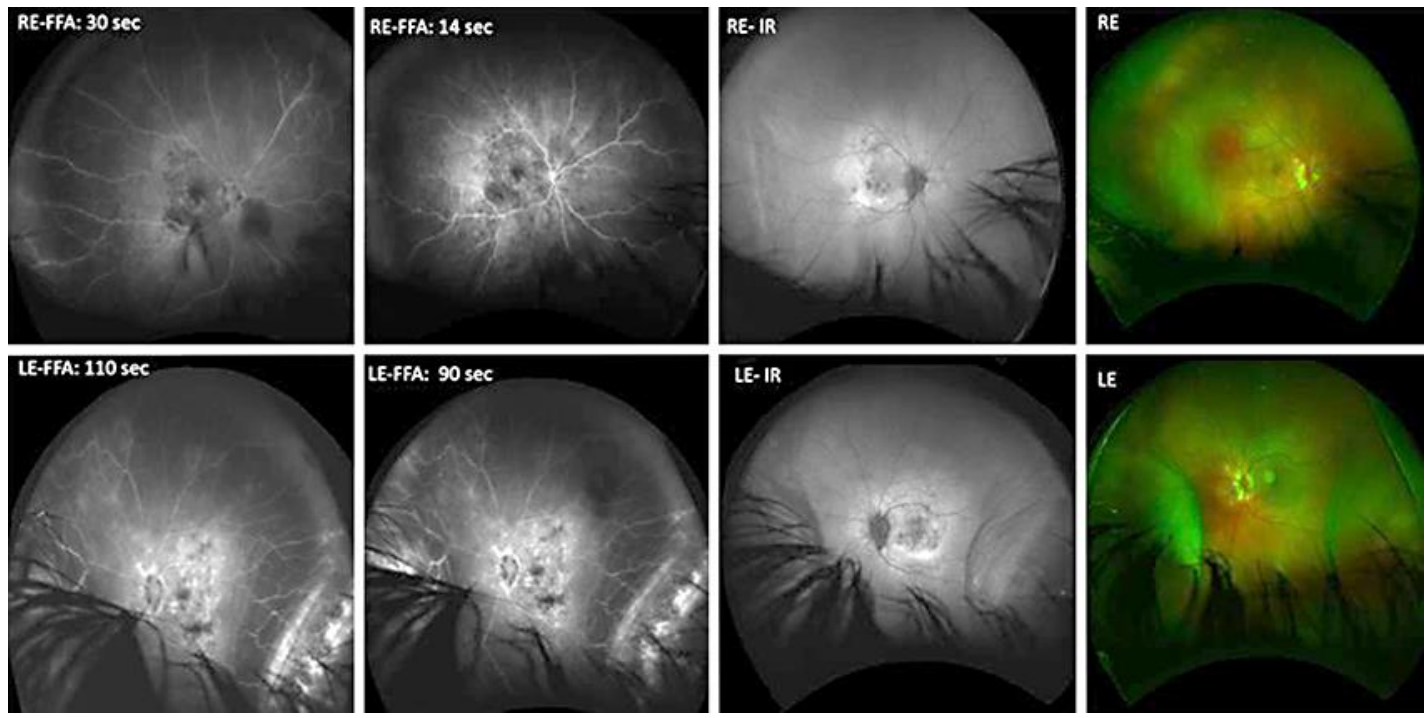

Fig. 2. Wide-field fundus imaging. Top: right eye (RE), bottom: left eye (LE). From right to left: wide-field color fundus photos and wide-field infrared (IR) fundus photos. Both modalities demonstrate choroidal elevation in both eyes. Fundus fluorescein angiography (FFA) shows late pooling of fluorescein peripherally.
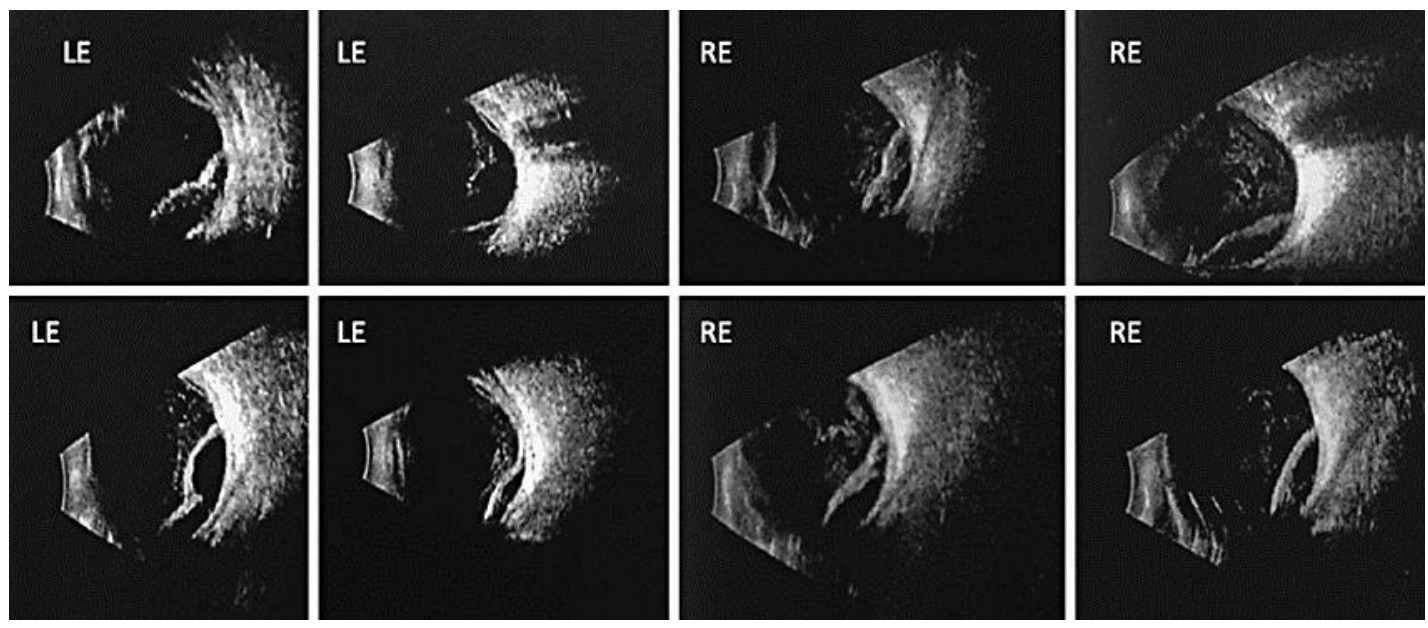

Fig. 3. B-ultrasonography of both right (RE) and left eye (LE) shows choroidal detachment in four quadrants. 


\section{Case Reports in Ophthalmology}
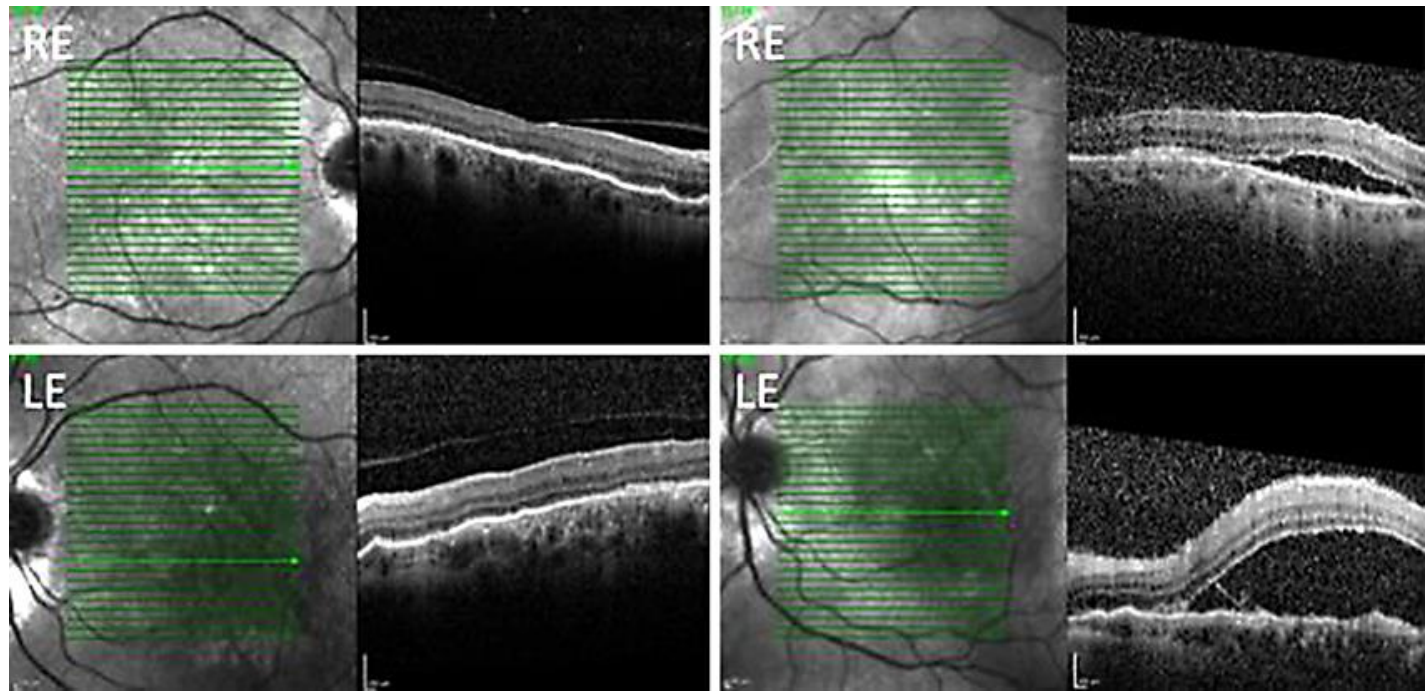

Fig. 4. Optical coherence tomography (OCT) scans of both right (RE) and left eye (LE). Both images on the right are of day 2. OCT shows subretinal fluid is more prominent in the LE. Images on the left are of 1 week post intravenous steroids with completed resolution of subretinal fluid but changes in the retinal pigment epithelium/photoreceptor complex.
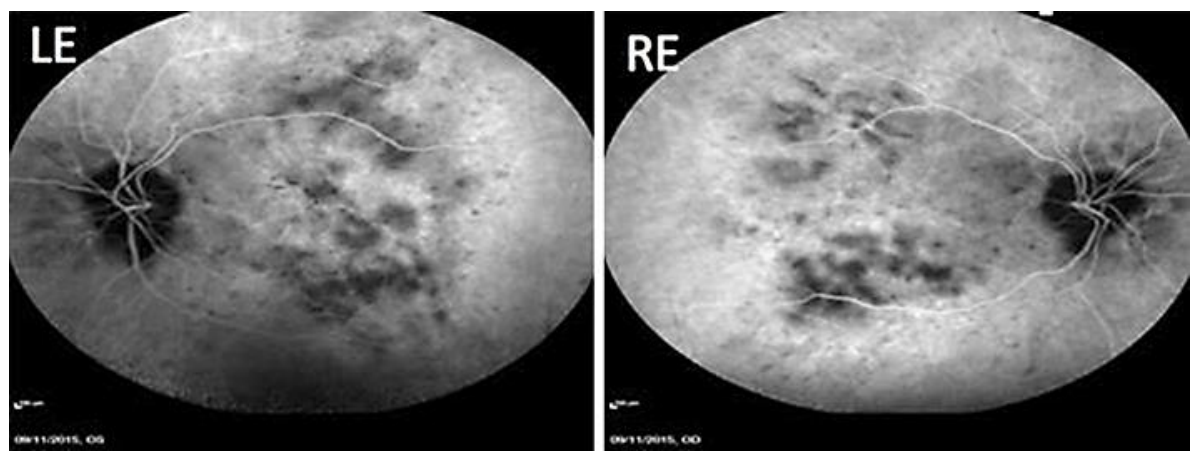

Fig. 5. Late indocyanine green chorioangiography of right (RE) and left (LE) eyes demonstrating multiple areas of choroidal hypoperfusion. 